\title{
Magnetism in galaxies - Observational overview and next generation radio telescopes
}

\author{
Rainer Beck \\ Max-Planck-Institut für Radioastronomie, Auf dem Hügel 69, 53121 Bonn, Germany \\ email: rbeck@mpifr-bonn.mpg.de
}

\begin{abstract}
The strength and structure of cosmic magnetic fields is best studied by observations of radio continuum emission, its polarization and its Faraday rotation. Fields with a well-ordered spiral structure exist in many types of galaxies. Total field strengths in spiral arms and bars are 20-30 $\mu \mathrm{G}$ and dynamically important. Strong fields in central regions can drive gas inflows towards an active nucleus. The strongest regular fields $(10-15 \mu \mathrm{G})$ are found in interarm regions, sometimes forming "magnetic spiral arms" between the optical arms. The typical degree of polarization is a few \% in spiral arms, but high (up to 50\%) in interarm regions. The detailed field structures suggest interaction with gas flows. Faraday rotation measures of the polarization vectors reveals large-scale patterns in several spiral galaxies which are regarded as signatures of large-scale (coherent) fields generated by dynamos. - Polarization observations with the forthcoming large radio telescopes will open a new era in the observation of magnetic fields and should help to understand their origin. Low-frequency radio synchrotron emission traces low-energy cosmic ray electrons which can propagate further away from their origin. LOFAR $(30-240 \mathrm{MHz})$ will allow us to map the structure of weak magnetic fields in the outer regions and halos of galaxies, in galaxy clusters and in the Milky Way. Polarization at higher frequencies $(1-10 \mathrm{GHz})$, to be observed with the EVLA, MeerKAT, APERTIF and the SKA, will trace magnetic fields in the disks and central regions of galaxies in unprecedented detail. All-sky surveys of Faraday rotation measures towards a dense grid of polarized background sources with ASKAP and the SKA are dedicated to measure magnetic fields in distant intervening galaxies and clusters, and will be used to model the overall structure and strength of the magnetic field in the Milky Way.
\end{abstract}

Keywords. Instrumentation: polarimeters, techniques: polarimetric, ISM: magnetic fields, galaxies: magnetic fields, galaxies: spiral, radio continuum: galaxies, radio continuum: ISM

\section{Introduction}

The amplification of cosmic fields from weak seed fields to the present-day level, their strength in intergalactic space, and their dynamical importance for galaxy evolution are important but unanswered astrophysical questions. Magnetic fields are believed to be a major agent in the ISM. They control the density and distribution of cosmic rays. Cosmic rays accelerated in supernova remnants can provide the pressure to drive galactic outflows and buoyant loops of magnetic fields via the Parker instability. Outflows from starburst galaxies in the early Universe may have magnetized the intergalactic medium.

\section{Origin of galactic magnetic fields}

Primordial seed fields of $10^{-20}-10^{-14} \mathrm{G}$ could originate from phase transitions in the early Universe (Widrow 2002, Caprini et al. 2009), from the time of cosmological structure formation by the Weibel instability (Lazar et al. 2009) or from injection by the first stars or jets generated by the first black holes (Rees 2005). High-energy $\gamma$-ray 
observations with HESS and FERMI indicate that the secondary particles are deflected by intergalactic fields of at least $10^{-16} \mathrm{G}$ strength with a filling factor larger than $60 \%$ (Dolag et al. 2010).

The most promising mechanism to sustain magnetic fields in the interstellar medium of galaxies is the dynamo (Beck et al. 1996). A small-scale dynamo in protogalaxies may have amplified seed fields to the energy density level of turbulence within less than $10^{8} \mathrm{yr}$ (Schleicher et al. 2010). To explain the generation of large-scale fields in galaxies, the mean-field dynamo has been developed. It is based on turbulence, differential rotation and helical gas flows ( $\alpha$-effect), generated by supernova explosions (Gressel et al. 2008, and this volume) and cosmic rays (Hanasz et al. 2009, and this volume). The meanfield dynamo in galaxy disks predicts that within a few $10^{9}$ yr large-scale regular fields are excited from the turbulent fields (Arshakian et al. 2009), forming spiral patterns (modes) with different azimuthal symmetries in the disk and vertical symmetries in the halo (Section 6).

The mean-field dynamo generates large-scale helicity with a non-zero mean in each hemisphere. As total helicity is a conserved quantity, the dynamo is quenched by the small-scale fields with opposite helicity unless these are removed from the system (Shukurov et al. 2006). Outflows are probably essential for effective mean-field dynamo action.

\section{Measuring magnetic fields in galaxies}

Magnetic fields need illumination to be detectable. Most of what we know about interstellar magnetic fields comes through the detection of radio waves. Zeeman splitting of radio spectral lines directly measures the field strength in gas clouds of the Milky Way (Heiles \& Troland 2005) and in starburst galaxies (Robishaw et al. 2008). The intensity of synchrotron emission is a measure of the number density of cosmic-ray electrons in the relevant energy range and of the strength of the total magnetic field component in the sky plane. Assuming energy equipartition between these two components allows us to calculate the total magnetic field strength from the synchrotron intensity (Section 4).

Polarized emission emerges from ordered fields in the sky plane. As polarization "vectors" are ambiguous by $180^{\circ}$, they cannot distinguish regular (coherent) fields, defined to have a constant direction within the telescope beam, from anisotropic fields, which are generated from turbulent fields by compressing or shearing gas flows and frequently reverse their direction within the telescope beam. Unpolarized synchrotron emission indicates turbulent (random) fields which have random directions in 3-D and have been amplified and tangled by turbulent gas flows.

The intrinsic degree of linear polarization of synchrotron emission is about $75 \%$. The observed degree of polarization is smaller due to the contribution of unpolarized thermal emission, which may dominate in star-forming regions, by Faraday depolarization along the line of sight and across the beam (Sokoloff et al. 1998), and by geometrical depolarization due to variations of the field orientation within the beam.

At long radio wavelengths, the polarization vector is rotated in a magnetized thermal plasma by Faraday rotation. If Faraday rotation is small (in galaxies typically at wavelengths shorter than a few centimeters), the observed $B$-vector gives the intrinsic field orientation in the sky plane, so that the magnetic pattern can be mapped directly. The rotation angle is proportional to the square of the wavelength $\lambda^{2}$ and to the Rotation Measure (RM), defined as the line-of-sight integral over the product of the plasma density and the strength of the field component along the line of sight. As the rotation angle is sensitive to the sign of the field direction, only regular fields give rise to Faraday rotation, while anisotropic and random fields do not. Measurements of the Faraday rotation 


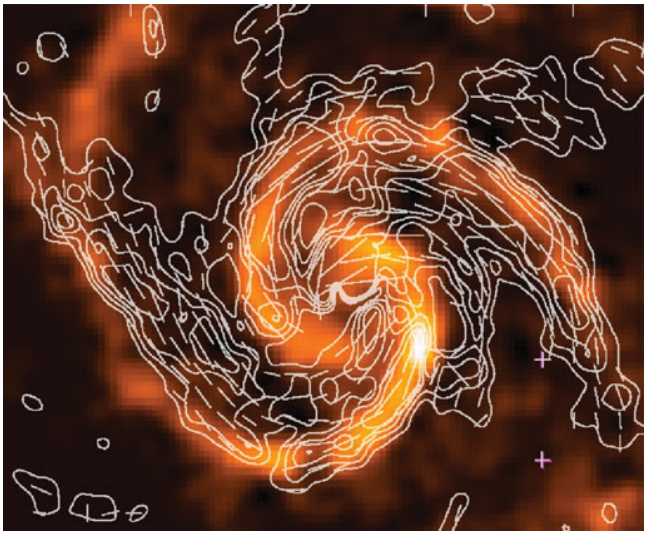

Figure 1. Polarized radio emission (contours) and $B$-vectors of M 51, combined from observations at $6 \mathrm{~cm}$ wavelength with the VLA and Effelsberg telescopes at to 8" resolution (Fletcher et al. 2010), overlaid onto an image of the $\mathrm{CO}(1-0)$ line emission by Helfer et al. (2003) (Copyright: MPIfR Bonn).

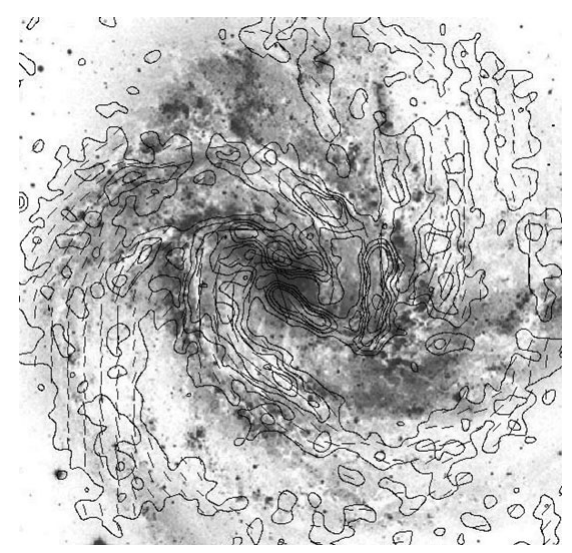

Figure 2. Polarized radio emission (contours) and $B$-vectors of $\mathrm{M} 83$, combined from observations at $6 \mathrm{~cm}$ wavelength with the VLA and Effelsberg telescopes and smoothed to 15" resolution (Beck, unpublished), overlaid onto an optical image from Dave Malin (Anglo Australian Observatory).

from multi-wavelength observations allow to determine the strength and direction of the regular field component along the line of sight. Dynamo modes of regular fields can be identified from the pattern of polarization angles and of RMs of the diffuse polarized emission of galaxy disks (Section 6).

Distinct emitting \& rotating regions located along the line of sight generate several RM components, and the observed average RM is a function of wavelength. In such cases, multi-channel spectro-polarimetric radio data are needed that can be Fourier-transformed into Faraday space, called RM Synthesis (Brentjens \& de Bruyn 2005). If the medium has a relatively simple structure, the 3 -D structure of the magnetized interstellar medium can be determined (Faraday tomography).

A grid of RM measurements of polarized background sources is another powerful tool to study magnetic field patterns in galaxies (Stepanov et al. 2008). A large number of background sources is required to recognize the field patterns, to separate the Galactic foreground contribution and to account for intrinsic RMs of the extragalactic sources.

\section{Total galactic magnetic fields}

The typical average equipartition strength of the total magnetic field (Beck \& Krause 2005) in spiral galaxies is about $10 \mu \mathrm{G}$, assuming energy equipartition between cosmic rays and magnetic fields. Radio-faint galaxies like M 31 and M 33, our Milky Way's neighbors, have weaker total magnetic fields (about $5 \mu \mathrm{G}$ ), while gas-rich spiral galaxies with high star-formation rates, like M 51 (Fig. 1), M 83 (Fig. 2) and NGC 6946 (Fig. 3), have total field strengths of $20-30 \mu \mathrm{G}$ in their spiral arms. The strongest total fields of 50-100 $\mu \mathrm{G}$ are found in starburst galaxies, like M 82 (Klein et al. 1988) and the "Antennae" NGC 4038/9 (Chyży \& Beck 2004), and in nuclear starburst regions, like in the center of NGC 1097 (Fig. 4) and of other barred galaxies (Beck et al. 2005).

If energy losses of cosmic-ray electrons are significant, especially in starburst regions or massive spiral arms, the equipartition values are lower limits (Beck \& Krause 2005). In starburst galaxies it is probably underestimated by a factor of a few (Thompson et al. 


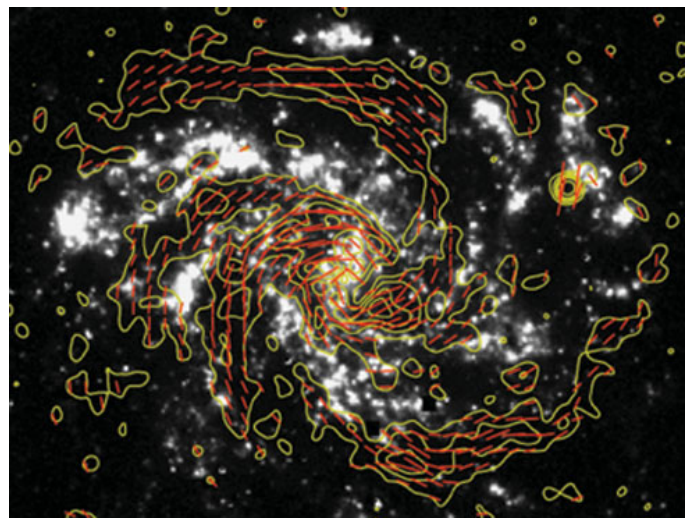

Figure 3. Polarized radio emission (contours) and $B$-vectors of NCC 6946 , combined from observations at $6 \mathrm{~cm}$ wavelength with the VLA and Effelsberg telescopes and smoothed to 15" resolution (Beck 2007), overlaid onto an $\mathrm{H} \alpha$ image by Ferguson et al. (1998) (Copyright: MPIfR Bonn; graphics: Sterne und Weltraum).

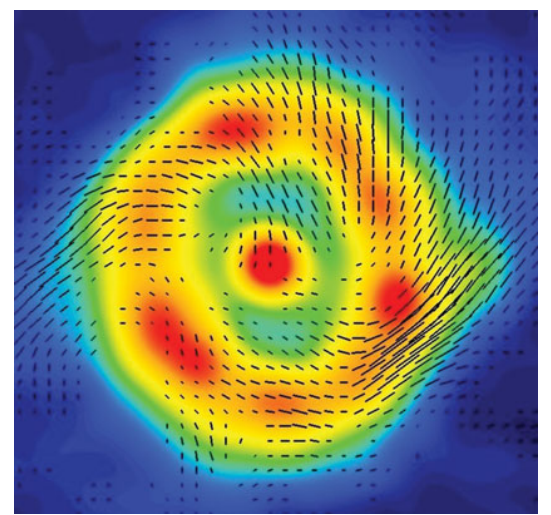

Figure 4. Total radio emission (contours) and $B$-vectors of the circum-nuclear ring of the barred galaxy NGC 1097, observed at $3 \mathrm{~cm}$ wavelength with the VLA and smoothed to 3" resolution (Beck et al. 2005) (Copyright: MPIfR Bonn).

2006). Field strengths of $0.5-18 \mathrm{mG}$ were detected in starburst galaxies by the Zeeman effect in the $\mathrm{OH}$ megamaser emission line at $18 \mathrm{~cm}$ (Robishaw et al. 2008).

The relative importance of various competing forces in the interstellar medium can be estimated by comparing the corresponding energy densities. The mean energy densities of the total (mostly turbulent) magnetic field and the cosmic rays in NGC 6946 and M 33 are $\simeq 10^{-11}$ erg cm$~^{-3}$ and $\simeq 10^{-12} \mathrm{erg} \mathrm{cm}^{-3}$, respectively (Beck 2007, Tabatabaei et al. 2008), similar to that of the turbulent gas motions across the whole star-forming disk, but about 10 times larger than that of the ionized gas (low-beta plasma). Magnetic fields are dynamically important.

The integrated luminosity of the total radio continuum emission at centimeter wavelengths (frequencies of a few $\mathrm{GHz}$ ), which is mostly of nonthermal synchrotron origin, and the far-infrared (FIR) luminosity of star-forming galaxies are tightly correlated. This correlation is one of the tightest correlations known in astronomy. It extends over five orders of magnitude (Bell 2003) and is valid in starburst galaxies to redshifts of at least 3 (Murphy 2009). Hence the total radio emission serves as a tracer of magnetic fields and of star formation out to large distances. The correlation requires that total (mostly turbulent) magnetic fields and star formation are connected, but the tightness needs multiple feedback mechanisms which are not yet understood (Lacki et al. 2010).

\section{Structure of ordered galactic magnetic fields}

Ordered (regular and/or anisotropic) field traced by the polarized synchrotron emission form spiral patterns in almost every galaxy, even in ring galaxies (Chyży \& Buta 2008), in flocculent galaxies without massive spiral arms (Soida et al. 2002), and in the central regions of galaxies and circum-nuclear gas rings (Fig. 4). Ordered fields are generally strongest $(10-15 \mu \mathrm{G})$ in the regions between the optical spiral arms and oriented parallel to the adjacent spiral arms. In some galaxies magnetic arms are formed, e.g. in IC 342 (Krause et al. 1993) and NGC 6946 (Fig. 3), with exceptionally high degrees of polarization (up to 50\%), possibly the result of higher dynamo modes (Section 6). In 


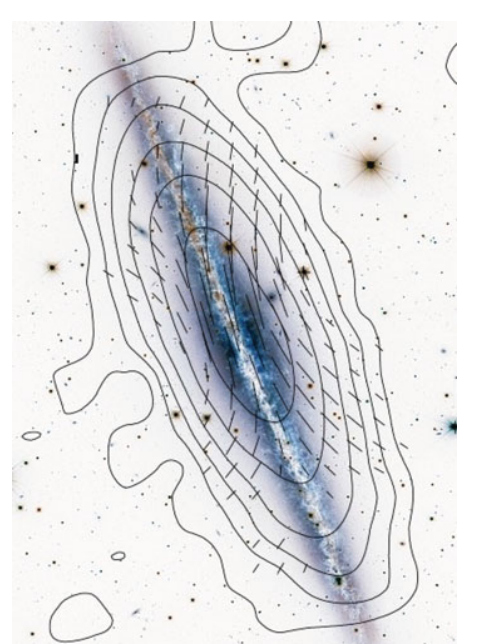

Figure 5. Total radio emission (84" resolution) and $B$-vectors of the edge-on galaxy NGC 891, a galaxy similar to the Milky Way, observed at $8.4 \mathrm{MHz}$ with the Effelsberg telescope (Krause 2009). The background optical image is from the CFHT. Copyright: MPIfR Bonn and CFHT/Coelum.

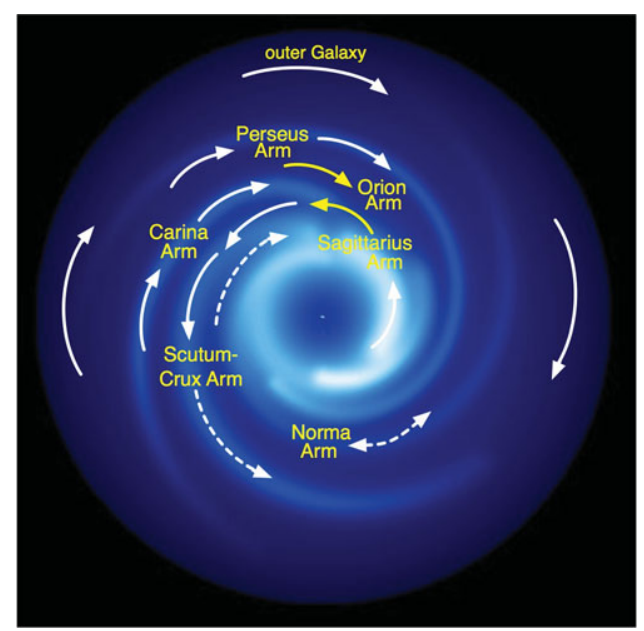

Figure 6. Model of the magnetic field in the Milky Way, derived from Faraday rotation measures of pulsars and extragalactic sources. Generally accepted results are indicated by yellow vectors, while white vectors are not fully confirmed (from Brown, priv. comm.).

galaxies with strong density waves like M 51 (Fig. 1) and M 83 (Fig. 2) enhanced ordered (anisotropic) fields occur at the inner edges of the inner optical arms, in the interarm regions and in the outer optical arms.

In galaxies with massive bars the field lines follow the gas flow. As the gas rotates faster than the bar pattern of a galaxy, a shock occurs in the cold gas which has a small sound speed, while the flow of warm, diffuse gas is only slightly compressed but sheared. The ordered field is also hardly compressed. It is probably coupled to the diffuse gas and strong enough to affect its flow (Beck et al. 2005). The polarization pattern in barred galaxies can be used as a tracer of shearing gas flows in the sky plane and hence complements spectroscopic measurements of radial velocities.

The central regions of galaxies are often sites of ongoing intense star formation and strong magnetic fields. NGC 1097 hosts a bright circum-nuclear ring with about $1.5 \mathrm{kpc}$ diameter (Fig. 4) and an active nucleus in its center. The ordered field in the ring has a spiral pattern and extends towards the nucleus. Magnetic stress in the circumnuclear ring due to the strong total magnetic field (about $50 \mu \mathrm{G}$ ) can drive gas inflow at a rate of several $\mathrm{M}_{\circ} / \mathrm{yr}$, which is sufficient to fuel the activity of the nucleus (Beck et al. 2005).

Nearby galaxies seen "edge-on" generally show a disk-parallel field near the disk plane. Edge-on galaxies like NGC 891 (Fig. 5) and NGC 253 (Heesen et al. 2009) reveal vertical field components in the halo forming an X-shaped pattern. The field is probably transported into the halo by an outflow emerging from the disk.

\section{Faraday rotation}

Spiral fields can be generated by compression at the inner edge of spiral arms, by shear in interarm regions, or by dynamo action (Section 2). Large-scale patterns of Faraday rotation measures (RM) are signatures of the mean-field dynamo and can be identified from the diffuse polarized emission of the galaxy disks (Krause 1990) or from RM data 
of polarized background sources (Stepanov et al. 2008). If several dynamo modes are superimposed, Fourier analysis of the RM variation is needed. The resolution of presentday observations is sufficient to identify not more than $2-3$ modes.

The disks of a few spiral galaxies reveal large-scale RM patterns. The Andromeda galaxy M 31 is the prototype of a dynamo-generated axisymmetric spiral disk field (Fletcher et al. 2004). Other candidates for a dominating axisymmetric disk field (dynamo mode $m=0$ ) are the nearby spiral IC 342 (Krause et al. 1989) and the irregular Large Magellanic Cloud (LMC) (Gaensler et al. 2005). Dominating bisymmetric spiral fields (dynamo mode $m=1$ ) are rare, which is also predicted by dynamo models. Faraday rotation in NGC 6946 and in other similar galaxies with magnetic arms can be described by a superposition of two azimuthal dynamo modes $(m=0$ and $m=2)$ (Beck 2007).

However, the spiral pattern of magnetic fields cannot be solely the result of mean-field dynamo action. If the beautiful spiral pattern of M 51 seen in radio polarization (Fig. 1) were only due to a regular field, its line-of sight component should generate a large-scale pattern in Faraday rotation, which is not observed. This means that a large amount of the ordered field is anisotropic and probably generated by compression and shear of the non-axisymmetric gas flows in the density-wave potential. The anisotropic field is strongest at the positions of the prominent dust lanes on the inner edge of the inner gas spiral arms, due to compression of turbulent fields in the density-wave shock. Regular fields (dynamo modes $m=0$ and $m=1$ ) also exist in the disk of M 51 but are much weaker than the anisotropic field (Fletcher et al. 2010).

In many other observed galaxy disks no clear patterns of Faraday rotation were found. Either several dynamo modes are superimposed and cannot be distinguished with the limited sensitivity and resolution of present-day telescopes, or the timescale for the generation of large-scale modes is longer than the galaxy's lifetime (Arshakian et al. 2009).

While the azimuthal symmetry of the magnetic field is known for many galaxies, the vertical symmetry (even or odd) is much harder to determine. The RM patterns of even and odd modes are similar in mildly inclined galaxies. The field of odd modes reverses its sign above and below the galactic plane. The symmetry type becomes only visible in strongly inclined galaxies, from the RM sign above and below the plane (Heesen et al. 2009), but the data are not yet conclusive.

\section{Magnetic fields in the Milky Way}

The detection of ultrahigh-energy cosmic rays (UHECRs) with the AUGER observatory and the anisotropic distribution of their arrival directions (Abreu et al. 2010) calls for a proper model of particle propagation. As UHECR particles are deflected by largescale regular fields and scattered by turbulent fields, the structure and extent of the fields in the disk and halo of the Milky Way need to be known.

The all-sky maps of polarized synchrotron emission at $1.4 \mathrm{GHz}$ from the Milky Way from DRAO and Villa Elisa and at 22.8 GHz from WMAP and the Effelsberg RM survey of polarized extragalactic sources were used to model the regular Galactic field (Sun et al. 2008). One large-scale field reversal is required at about 1-2 kpc from the Sun towards the Milky Way's center (Fig. 6), which is also supported by the detailed study of RMs from extragalactic sources near the Galactic plane (Van Eck et al. 2010). More large-scale reversals possibly exist (Han et al. 2006).

In mildly inclined spiral galaxies, no large-scale field reversals at certain radial distances from a galaxy's center have yet been detected, although high-resolution RM maps are available for many galaxies. The reversals in the Milky Way may be of limited azimuthal extent, and then are difficult to observe in external galaxies with the resolution of present- 
day telescopes. Alternatively, the reversals in the Milky Way may be part of a disturbed field structure, e.g. due to interaction with the Magellanic clouds.

The signs of RMs of extragalactic sources and of pulsars at Galactic longitudes $1=90^{\circ}$ $270^{\circ}$ are the same above and below the plane (Taylor et al. 2009): the local magnetic field is symmetric, while the RM signs towards the inner Galaxy $\left(1=270^{\circ}-90^{\circ}\right)$ are opposite above and below the plane. This can be assigned to an antisymmetric halo field (Sun et al. 2008) or to deviations of the local field (Wolleben et al. 2010). In conclusion, the overall structure of the regular field in the disk of the Milky Way is not known yet - its structure cannot be described by a simple large-scale pattern (Noutsos 2009). A larger sample of pulsar and extragalactic RM data is needed.

Little is known about the halo field in the Milky Way. The synchrotron scale height of about $1.5 \mathrm{kpc}$ indicates a scale height of the total field of at least $6 \mathrm{kpc}$. The local regular Galactic field, according to RM data from extragalactic sources, has no significant vertical component towards the northern Galactic pole and only a weak vertical component of $B_{z} \simeq 0.3 \mu \mathrm{G}$ towards the south (Mao et al. 2010).

\section{Outlook}

High-resolution, high-sensitivity observations at high frequencies with the planned Square Kilometre Array (SKA) will directly map the detailed field structure and the interaction with the gas. Synchrotron emission, signature of total magnetic fields, can be detected with the SKA out to very large redshifts for starburst galaxies, depending on luminosity and magnetic field strength (Murphy 2009). If the emission from galaxies is too weak to be detected, the method of RM grids towards background QSOs can still be applied. Here, the distance limit is given by the polarized flux of the background QSO which can be much higher than that of the intervening galaxy. Regular fields of several $\mu \mathrm{G}$ strength were already detected in distant galaxies (Bernet et al. 2008). Mean-field dynamo theory predicts RMs from evolving regular fields with increasing coherence scale at $z \leqslant 3$ (Arshakian et al. 2009).

The SKA "Magnetism" Key Science Project plans to observe an all-sky survey (at least $10^{4} \mathrm{deg}^{2}$ ) around $1 \mathrm{GHz}$ which will measure at least $1500 \mathrm{RMs}$ per square degree, in total at least $2 \times 10^{7} \mathrm{RMs}$ from compact polarized extragalactic sources at a mean spacing of $\simeq 90^{\prime \prime}$ and at least 20000 RMs from pulsars (Gaensler et al. 2004). This will allow the detailed reconstruction of the 3-D field structure in the Milky Way and in many nearby galaxies, while simple patterns of regular fields can be recognized out to distances of about $100 \mathrm{Mpc}$ (Stepanov 2008). All-sky and deep-field RM surveys are also planned with the SKA precursor telescopes, ASKAP (Gaensler et al. 2010) and MeerKAT, and with the pathfinder telescope APERTIF.

The recently completed low-frequency radio telescopes Low Frequency Array (LOFAR) and the Murchison Widefield Array (MWA) (under construction) are suitable instruments to search for extended synchrotron radiation at the lowest possible levels in outer galaxy disks and halos and the transition to intergalactic space. Low frequencies are also ideal to search for small Faraday rotation measures from weak interstellar and intergalactic fields (Beck 2009) and in galaxy clusters (see Feretti and Dolag, this volume).

\section{References}

Abreu, P. \& The Pierre Auger Collaboration 2010, Astroparticle Physics, 34, 314

Arshakian, T. G., Beck, R., Krause, M., \& Sokoloff, D. 2009, A $\& A, 494,21$

Beck, R. 2007, A\& A, 470, 539 
Beck, R. 2009, Rev. Mex. AyA, 36, 1

Beck, R. \& Hoernes, P. 1996, Nature, 379, 47

Beck, R. \& Krause, M. 2005, AN, 326, 414

Beck, R., Brandenburg, A., Moss, D., Shukurov, A., \& Sokoloff, D. 1996, ARAA, 34, 155

Beck, R., Fletcher, A., Shukurov, A., et al. 2005, A\&A, 444, 739

Bell, E. F. 2003, ApJ, 586, 794

Bernet, M. L., Miniati, F., Lilly, S. J., Kronberg, P. P., \& Dessauges-Zavadsky, M. 2008, Nature, 454,302

Brentjens, M. A. \& de Bruyn, A. G. 2005, A\&A, 441, 1217

Caprini, C., Durrer, R., \& Fenu, E. 2009, J. Cosmology \& Astroparticle Physics, 11(2009)001

Chyży, K. T. 2008, A\&̈A, 482, 755

Chyży, K. T. \& Beck, R. 2004, A\& A, 417, 541

Chyży, K. T. \& Buta, R. J. 2008, ApJ, 677, L17

Dolag, K., Kachelriess, M., Ostapchenko, S., \& Tomas, R. 2010, arXiv:1009.1782

Ferguson, A. M. N., Wyse, R. F. G., Gallagher, J. S., \& Hunter, D. A. 1998, ApJ, 506, L19

Fletcher, A., Berkhuijsen, E. M., Beck, R., \& Shukurov, A. 2004, A\&A, 414, 53

Fletcher, A., Beck, R., Shukurov, A., Berkhuijsen, E. M., \& Horellou, C. 2010, MNRAS, in press

Gaensler, B. M., Beck, R., \& Feretti, L. 2004, New Astr. Revs, 48, 1003

Gaensler, B. M., Haverkorn, M., Staveley-Smith, L., et al. 2005, Science, 307, 1610

Gaensler, B. M., Landecker, T. L., \& Taylor, A. R. 2010, BAAS, 42, 470

Gressel, O., Elstner, D., Ziegler, U., \& Rüdiger, G. 2008, A\&SA, 486, L35

Han, J. L., Manchester, R. N., Lyne, A. G., Qiao, G. J., \& van Straten, W. 2006, ApJ, 624, 868

Hanasz, M., Wóltański, D., \& Kowalik, K. 2009, ApJ, 706, L155

Heesen, V., Krause, M., Beck, R., \& Dettmar, R.-J. 2009, A\&广A, 506, 1123

Heiles, C. \& Troland, T. H. 2005, ApJ, 624, 773

Helfer, T. T., Thornley, M. D., Regan, M. W., et al. 2003, ApJS, 145, 259

Klein, U., Wielebinski, R., \& Morsi, H. W. 1988, A\&A, 190, 41

Krause, M. 1990, in: R. Beck et al. (eds.), Galactic and Intergalactic Magnetic Fields (Dordrecht: Kluwer), p. 187

Krause, M. 1993, in: F. Krause et al. (eds.), The Cosmic Dynamo (Dordrecht: Kluwer), p. 303

Krause, M. 2009, Rev. Mex. AyA, 36, 25

Krause, M., Hummel, E., \& Beck, R. 1989, A\&SA, 217, 4

Lacki, B. C., Thompson, T. A., \& Quataert, E. 2010, ApJ, 717, 1

Lazar, M., Schlickeiser, R., Wielebinski, R., \& Poedts, S. 2009, ApJ, 693, 1133

Mao, S. A., Gaensler, B. M., Haverkorn, M., et al. 2010, ApJ, 714, 1170

Murphy, E. 2009, ApJ, 706, 482

Noutsos, A. 2009, in: K. G. Strassmeier et al. (eds.), Cosmic Magnetic Fields: From Planets, to Stars and Galaxies (Cambridge: Cambridge Univ. Press), p. 15

Rees, M. J. 2005, in: R. Wielebinski \& R. Beck (eds.), Cosmic Magnetic Fields (Berlin: Springer), p. 1

Robishaw, T., Quataert, E., \& Heiles, C. 2008, ApJ, 680, 981

Schleicher, D. R. G., Banerjee, R., Sur, S., et al. 2010, A\&SA, 522, A115

Shukurov, A., Sokoloff, D., Subramanian, K., \& Brandenburg, A. 2006, A\& A, 448, L33

Soida, M., Beck, R., Urbanik, M., \& Braine, J. 2002, A\&A, 394, 47

Sokoloff, D. D., Bykov, A. A., Shukurov, A., et al. 1998, MNRAS, 299, 189, and Erratum in MNRAS, 303, 207

Stepanov, R., Arshakian, T. G., Beck, R., Frick, P., \& Krause, M. 2008, A\&3A, 480, 45

Sun, X. H., Reich, W., Waelkens, A., \& Enßlin, T.A. 2008, $A \mathscr{E} A, 477,573$

Tabatabaei, F., Krause, M., Fletcher, A., \& Beck, R. 2008, A\&A, 490, 1005

Taylor, A. R., Stil, J. M., \& Sunstrum, C. 2009, ApJ, 702, 1230

Thompson, T. A., Quataert, E., Waxman, E., Murray, N., \& Martin, C. L. 2006, ApJ, 645, 186

Van Eck, C. L., Brown, J. C., Stil, J. M., et al. 2010, ApJ, in press

Widrow, L. M. 2002, Rev. Mod. Phys., 74, 775

Wolleben, M., Fletcher, A., Landecker, T. L., et al. 2010, ApJ, 724, L48 